\title{
An energy-based failure criterion for delamination initiation in
}

\section{electronic packaging}

HAIBO FAN ${ }^{1 *}$, PAULAR W.K. CHUNG ${ }^{1}$, MATTHEW M.F. YUEN ${ }^{1}$, and PHILIP C.H. CHAN ${ }^{2}$

${ }^{I}$ Department of Mechanical Engineering, ${ }^{2}$ Department of Electrical and Electronic Engineering,

Hong Kong University of Science and Technology, Clear Water Bay, Kowloon, Hong Kong SAR, China

\begin{abstract}
The significance of interfacial delamination as a crucial failure mechanism in electronic packaging has been documented in many papers. A number of failure criteria have been used to solve the problems with a pre-crack at the interface. However, in real electronic packages, the size and location of the cracks or/and delamination cannot be predicted. It is not easy to use the traditional fracture criteria to deal with more complicated 3-D delamination problems. The interface of epoxy molding compound (EMC)/copper leadframe was selected in the study. A series of button shear tests were conducted to evaluate the interfacial adhesion between EMC and copper. In each test, the failure load acting on the EMC of the button shear sample was measured at different shear angles and a finite element model was used to evaluate the stresses at the EMC/copper interface. In this paper, an energy-based failure criterion is proposed by deriving the interfacial strain energy density to the tensile and shear failure modes across the chosen EMC/copper interface. Stresses were extracted from the numerical simulation in order to calculate the distortional interfacial strain energy density, $U_{d}$, and the hydrostatic interfacial strain energy density, $U_{h}$, related, respectively, to the shear and tensile modes. $U_{d}$ and $U_{h}$ were averaged within a selected region of the finite element model where it exhibits high interfacial strain energy density values.
\end{abstract}

Key words: Delamination, failure criterion, button shear test, and finite element method.

\footnotetext{
* To whom correspondence should be addressed: Tel: (852)-2358 8814; Fax: (852) 2358 1202;

E-mail: mehaibo@ust.hk,
} 


\section{Introduction}

With the increasing need for high input/output $(\mathrm{I} / \mathrm{O})$ counts and miniaturization, novel electronic packages are continuously being developed. Interfacial failure is an important issue in electronic packages and assemblies due to their multilayered structure. It is known that the mismatch of the coefficient of thermal expansion between the different layers in electronic packages could result in high interfacial stresses. Delamination occurring during the manufacture will propagate though the interface and destroy the functionality of the device.

Delamination prediction is still a challenge both from the scientific and industrial points of view because of the large number of parameters and the complex state of stresses involved in the design of laminated structures. Several accepted methodologies on how to quantify the interfacial strength and predict interfacial delamination have been established. Generally, two different failure criteria have been commonly employed to quantify the interfacial strength and predict interfacial delamination in laminates and IC packages. One of them is based on the fracture mechanics approach, where an interface crack propagates when the interface stress intensity factor or energy release rate exceeds a critical value [1]. This approach is useful for analyzing the reliability and life of a product that is assumed to contain some inherent cracks. However, the amount, location and size of delamination or crack cannot be easily determined in real packages, thus it poses a problem for predicting failure at interfaces in IC packages.

The other criterion is the strength-based approach, where some predefined critical loads or average stresses are utilized to assess the interface strength [2-5]. In this approach, the maximum load that materials or structure can sustain before interface failure is measured by applying tensile or shear loads to the test specimens. It is relative easy to perform experiments based on the strength-based approach and the failure criterion developed on the 
basis of such tests should be very useful for design of products and the prediction of product reliability.

Among of the strength-based approaches, strain energy density based failure criterion assumes that failure occurs when the strain energy density at any location at the interface exceeds a limiting value. This limiting value can be defined as the area under a tensile or shear stress-strain curve. This criterion was introduced by Shih and Macdonald [6] and later applied to an extensive class of problems by Shih and co-workers [7-8]. Based on an ideal elastic-plastic and bilinear plastic model, Tong, et al [9] proposed a simple formula to express the failure load of an adhesive bonded double lap joint in terms of the strain energy density.

The button shear test, which is widely used in industry, was conducted to evaluate the bi-material properties. Much work has been conducted using this test method to evaluate the adhesion with different preconditions. Using the button shear test, an allowable energy is evaluated for the initiation of delamiantion at the EMC and copper interface, which has been successfully adopted by Fan and coworkers [10-13].

In the present study, the angular button shear test was conducted to evaluate the interfacial adhesion between the EMC and copper. Finite element modeling of the button shear test samples was conducted to obtain interfacial stresses, and the average interfacial strain energy density was then calculated. The average interfacial strain energy densities at different shear angles were used to determine a critical value for the EMC/copper interface, which could be incorporated into a strength-based failure criterion for interfacial delamination. The failure criterion was verified experimentally with five different EMC materials. 


\section{Button Shear Test}

\subsection{Sample preparation and test configuration}

The button shear test, which is widely used in industry, was conducted to evaluate the bi-material properties between EMC and copper leadframe. The leadframe material investigated had $99.8 \%$ copper. Five commercial EMC materials selected for this study were HC-100-XGB (EMC 1), MP-7250H-3 (EMC 2), HC-100XJA (EMC 3), HC-10-2 (EMC 4) and MP-8000C (EMC 5), which all were supplied by Nitto Denko (HK) Co. Ltd..

Copper leadframe strips of $13 \mathrm{~mm}$ width and $70 \mathrm{~mm}$ length were prepared for the study (a schematic diagram is shown in Fig. 1). The copper leadframe strip was degreased, and cleaned using oxide cleaning agents. The button-shape EMC was then molded on the cleaned copper strip and cured. The curing process followed the specifications of manufacturers.

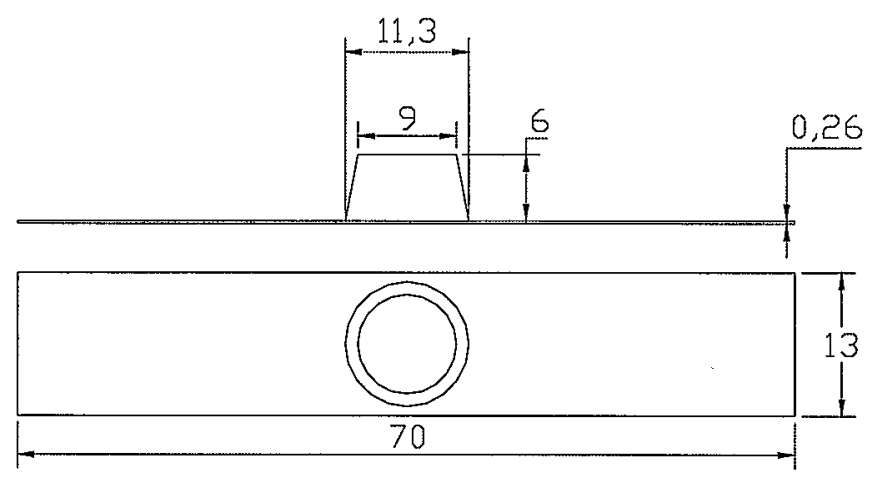

Figure 1. The dimensions (in $\mathrm{mm}$ ) of the button shear test sample.

Shear tests were conducted using a Dage 4000 shear tester with a DS100 load cell. The button shear test specimen was fixed on the mounting jig attached onto the shear tester platform. A special test jig was designed and made for clamping the sample and testing with preset angular loadings of $-10^{\circ}, 0^{\circ}, 10^{\circ}$ and $20^{\circ}$. A schematic drawing of the angular jig set up is shown in Fig. 2. 
A load with a loading rate of $85 \mu \mathrm{m} / \mathrm{s}$ was applied to the button surface with a controlled loading height, as shown in Fig. 2. The breaking load of the specimen with interfacial fracture was recorded. Five samples were tested for each loading angle setting. All experiments were conducted at room temperature.

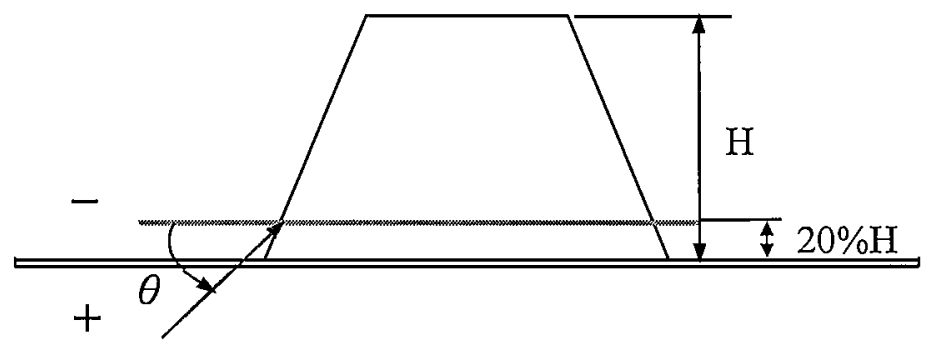

Figure 2. The schematic drawing of the angular shear test.

\subsection{Experimental results}

The specimens were divided into five groups with five different EMC materials and the same copper material. In each group, four different shear angular loadings were applied in the shear test for a given material system. A typical load-displacement graph was obtained from the angular button shear test as shown in Fig. 3. It can be seen that the loaddisplacement curve is fairly linear until the maximum load is reached. The fracture surface of each sample was inspected visually after the button shear test. Every test sample in the button shear test shows a clean substrate surface after debonding, confirming an interfacial failure which means the strength at the interface was less than the bulk strength of either the EMC or copper substrate. The average failure forces obtained with different angles for the five materials are shown in Fig. 4. As expected, the failure forces become smaller with angle varying from $-10^{\circ}$ to $20^{\circ}$ for all EMC materials. The negative shear angle gives a more compressive load to the $\mathrm{EMC} / \mathrm{copper}$ interface, so a higher failure force is needed to shear the 
button off from the copper substrate. The failure load is inversely proportional to the shear angle.

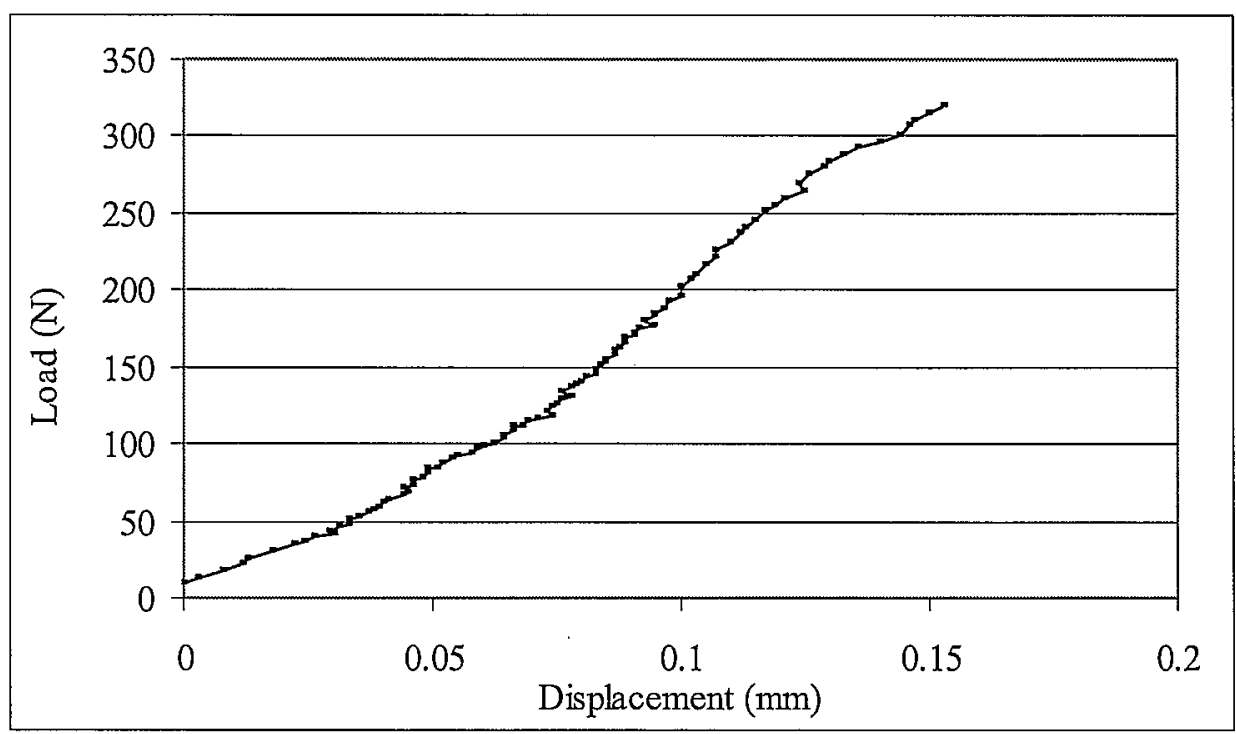

Figure 3. Typical load-displacement curve from the button shear test.



Figure 4. Average failure forces for different angles for five EMC materials. 


\section{Finite Element Model}

In order to predict the interfacial delamination between EMC and copper substrate under mechanical loading, it is necessary to perform a finite element analysis to calculate the interfacial stresses and to evaluate the critical interfacial strain energy density based on experimental results from the button shear tests. The ANSYS code was employed in the stress modeling of all test specimens. This paper focuses on the delamination at the EMC/copper interface. The materials are assumed to be linear elastic, homogeneous and isotropic. The Young's modulus and Poisson's ratios of the materials are listed in Table 1.

Table 1.

Material properties of EMC materials and $\mathrm{Cu}$.

\begin{tabular}{|c|c|c|}
\hline Properties & $\begin{array}{c}\text { Young's Modulus } \\
(\mathrm{GPa})\end{array}$ & Poisson's Ratio \\
\hline EMC 1 & 18.2 & 0.3 \\
\hline EMC 2 & 14.2 & 0.3 \\
\hline EMC 3 & 23.8 & 0.3 \\
\hline EMC 4 & 13.3 & 0.3 \\
\hline EMC 5 & 17.2 & 0.3 \\
\hline Cu & 110 & 0.36 \\
\hline
\end{tabular}

A three-dimensional finite element model was built to calculate the stresses at the EMC/copper interface. Eight-node solid element was used. The global finite element meshing is given in Fig. 5. The mesh was refined at the edges and the interface to capture the steep stress gradients expected as shown in Fig. 6. The elements varied in sizes from $0.06 \mathrm{x}$ 
$0.12 \times 0.08 \mathrm{~mm}$ near the interface edge to $0.8 \times 0.05 \times 0.1 \mathrm{~mm}$ near the interface center. The mesh densities ensure that the stress distribution at the interface remains unchanged with further refinement of the meshes. Boundary conditions and loading were applied to represent the test conditions in the button shear test. To simulate the constraint of the button shear specimen, the nodes at the substrate surface were all fixed.

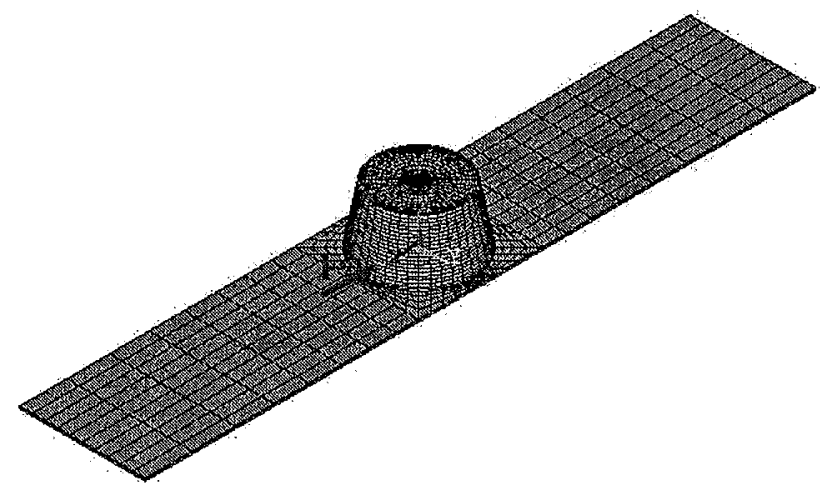

Figure 5. Finite element model of the button shear test



(a)

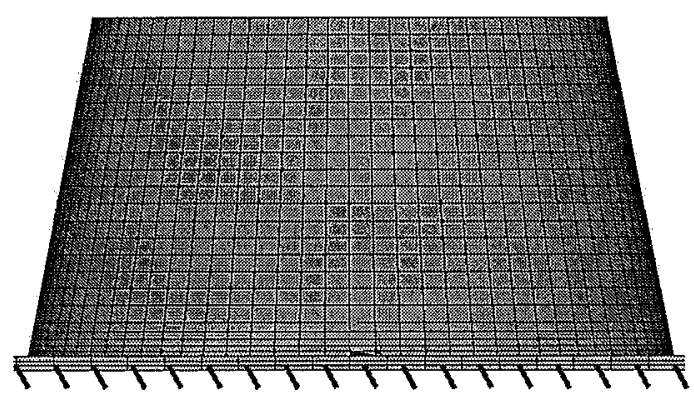

(b)

Figure 6. Top (a) and front (b) views of the meshed button and substrate of the finite element model.

The failure load for each test specimen was used in the finite element model for the determination of the allowable interfacial strain energy density. There exist complex stress 
states and stress concentrations at the loading edge of the EMC/copper interface, and the interfacial tensile stress along the interface from the loading edge is shown in Fig. 7 using $d$ as a distance from the loading edge. The interfacial tensile stress is greatest at the loading edge directly under the applied shear load, and it confirms that delamination will initiate at this point.

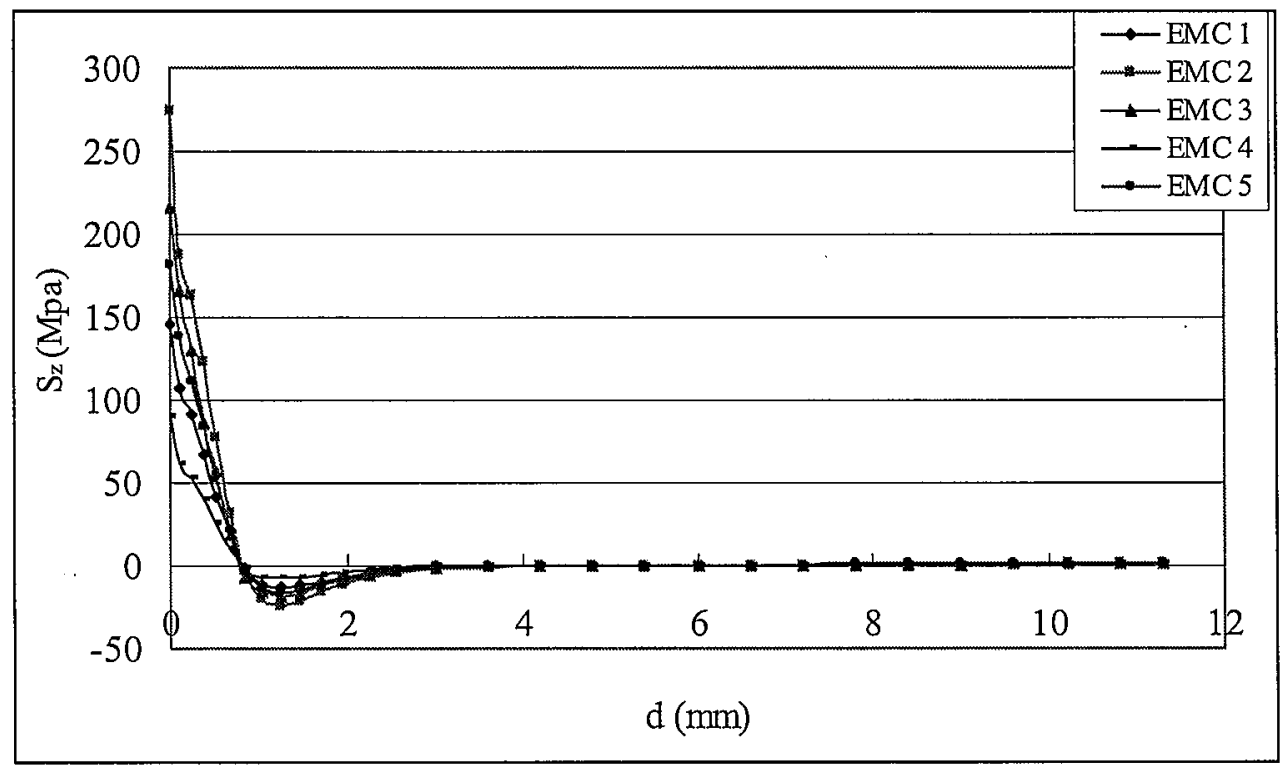

Figure 7. The interfacial tensile stress $\left(\mathrm{S}_{\mathrm{Z}}\right)$ along the EMC/copper interface

\section{Interfacial Delamination Failure Criterion}

In order to predict the interfacial delamination initiation and growth, it is necessary to consider the stresses and strains across the EMC/copper interface for all button shear specimen test results. Several previously proposed interfacial failure criteria are based on the strength-based approach. Sun and Zhou [14] and Kim and Soni [15] have proposed criteria to predict interfacial delamination at the free edges of composite laminates based on averaging of interfacial stresses. Shih [8] developed the strain energy density (SED) failure criterion 
that investigated the local strain energy density in the region of interest. This criterion has been successfully used in two- and three-dimensional crack problems, dynamic crack problems, failure initiating from notches, ductile fracture involving prediction of crack initiation, slow stable crack growth and final separation, etc. For a linear elastic material behavior, the total strain energy density and distortional strain energy density at various locations can be obtained using equation (1) and equation (2), respectively. The hydrostatic strain energy density can be expressed as equation (3).

$$
\begin{gathered}
U=\frac{1}{2 E}\left[\sigma_{x}^{2}+\sigma_{y}^{2}+\sigma_{z}^{2}-2 \psi\left(\sigma_{x} \sigma_{y}+\sigma_{y} \sigma_{z}+\sigma_{z} \sigma_{x}\right)\right]+\frac{1+\gamma}{E}\left[\tau_{x y}^{2}+\tau_{y z}^{2}+\tau_{z x}^{2}\right] \\
U_{d}=\frac{1+v}{6 E}\left[\left(\sigma_{x}-\sigma_{y}\right)^{2}+\left(\sigma_{y}-\sigma_{z}\right)^{2}+\left(\sigma_{z}-\sigma_{x}\right)^{2}+6\left(\tau_{x y}^{2}+\tau_{y z}^{2}+\tau_{z x}^{2}\right)\right] \\
U_{h}=U-U_{d}
\end{gathered}
$$

where $U, U_{d}$ and $U_{h}$ are the total, distortional and hydrostatic strain energies per unit volume respectively; $\sigma$ and $\tau$ are the stress components in the $\mathrm{x}, \mathrm{y}$ and $\mathrm{z}$ directions; $E$ is Young's modulus; $v$ is Poisson's ratio.

The distribution of interfacial strain energy density at the EMC/copper interface is illustrated in Fig. 8. It can be seen that the interfacial strain energy density is highest at the loading edge directly under the shear load. The interfacial delamination between the EMC and copper is mainly governed by the interfacial strain energy density distribution at this location. This also confirms that delamination will occur at the loading edge of the interface directly under the shear load. 


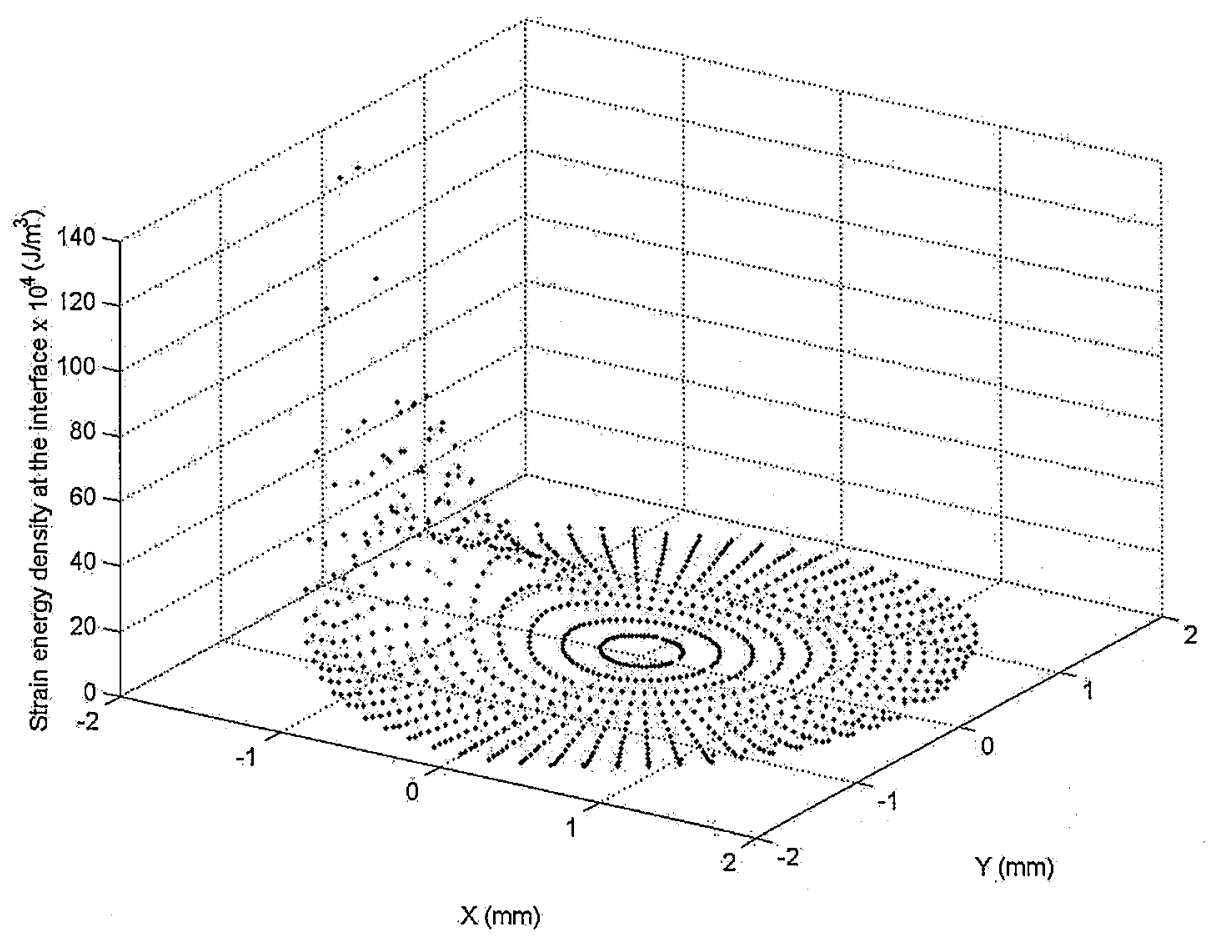

Figure 8. Distribution of interfacial strain energy density at the EMC/copper interface.

Due to the singular nature of the interfacial strain energy density at the loading edge, an averaging approach was used for deducing the interfacial delamination criterion taking into account both the distortional and hydrostatic strain energy densities. In this approach, the average interfacial strain energy density is obtained by applying the normalized volumetric weighting to the strain energy density for each of the elements at the EMC/copper interface. The average interfacial strain energy density can be written as:

$$
\bar{U}=\frac{\sum_{i} U_{i} \cdot V_{i}}{\sum_{i} V_{i}}
$$

where $\bar{U}$ is the average interfacial strain energy density for all the interfacial elements, $U_{i}$ is the interfacial strain energy density of the $\mathrm{i}$-th element, and $V_{i}$ is the volume of the $\mathrm{i}$-th 
element. According to the above formula, the average distortional and hydrostatic strain energy densities are $\bar{U}_{d}$ and $\bar{U}_{h}$, respectively.

Across the interface, the interfacial strain energy density criterion states that delamination for the particular bi-material system will initiate at the interface if the average value of interfacial strain energy density across the interface exceeds a certain critical value. Hunt and Carlsson [4] investigated debonding in overmolded integrated packages using an average stress approach and discussed the choice of the region for averaging. Using theri approach in this study, two adjacent layers of interfacial elements, one on each side of the EMC and copper interface, were selected within a region of radius $0.26 \mathrm{~mm}$ centered at the point on the loading edge directly under the shear load. The chosen radius is the same as the leadframe thickness of the button shear specimen. Under different loading conditions, the average distortional interfacial strain energy density $\bar{U}_{d}$ and hydrostatic interfacial strain energy density $\bar{U}_{h}$ were calculated for the different EMC/copper material systems. The results for the five EMC materials are shown in Fig. 9. Clearly, it can be seen that $\bar{U}_{d}$ decreases and $\bar{U}_{h}$ increases with the shear angle varying from $-10^{\circ}$ to $20^{\circ}$ and $\bar{U}_{d}$ is nearly linear with $\bar{U}_{h}$ for each EMC material. The magnitude of $\bar{U}_{d}$ is higher than that of $\bar{U}_{h}$. It can be concluded that $\bar{U}_{d}$ has more contribution to interfacial delamination when the shear angle is $-10^{\circ}$ and, on the contrary, $\bar{U}_{h}$ has more contribution to the interfacial delamination as the shear angle becomes larger. Because the critical value of $\bar{U}_{h}$ at the interfacial edge is much lower than that of $\vec{U}_{d}$, the energy required for interfacial delamination becomes rather small as the shear angle becomes larger. Likewise the failure load will become smaller with a larger shear angle. 


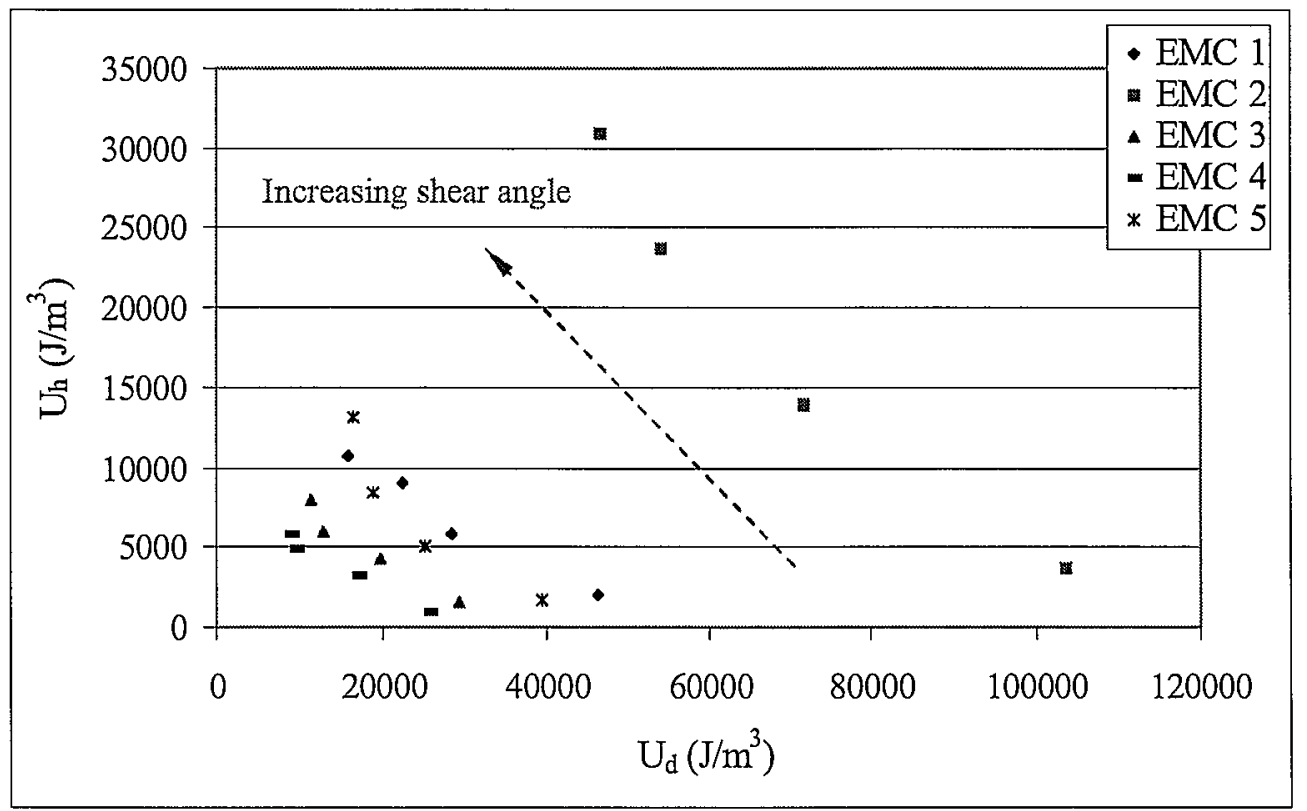

Figure 9. Average strain energy densities $U_{d}$ vs $U_{b}$ for the 5 EMC materials

Based on the above results, $\bar{U}_{d}$ and $\bar{U}_{h}$ are used here as two failure parameters to predict interfacial delamination. Interfacial strain energy density criterion is given as follow:

$$
\frac{\bar{U}_{d}}{U_{d c}}+\frac{\overline{U_{h}}}{U_{h c}}=1
$$

where $U_{d c}$ and $U_{h c}$ are, respectively, the critical distortional interfacial strain energy density under pure shear and the hydrostatic interfacial strain energy density under hydrostatic pressure, which are properties of the particular interface. 


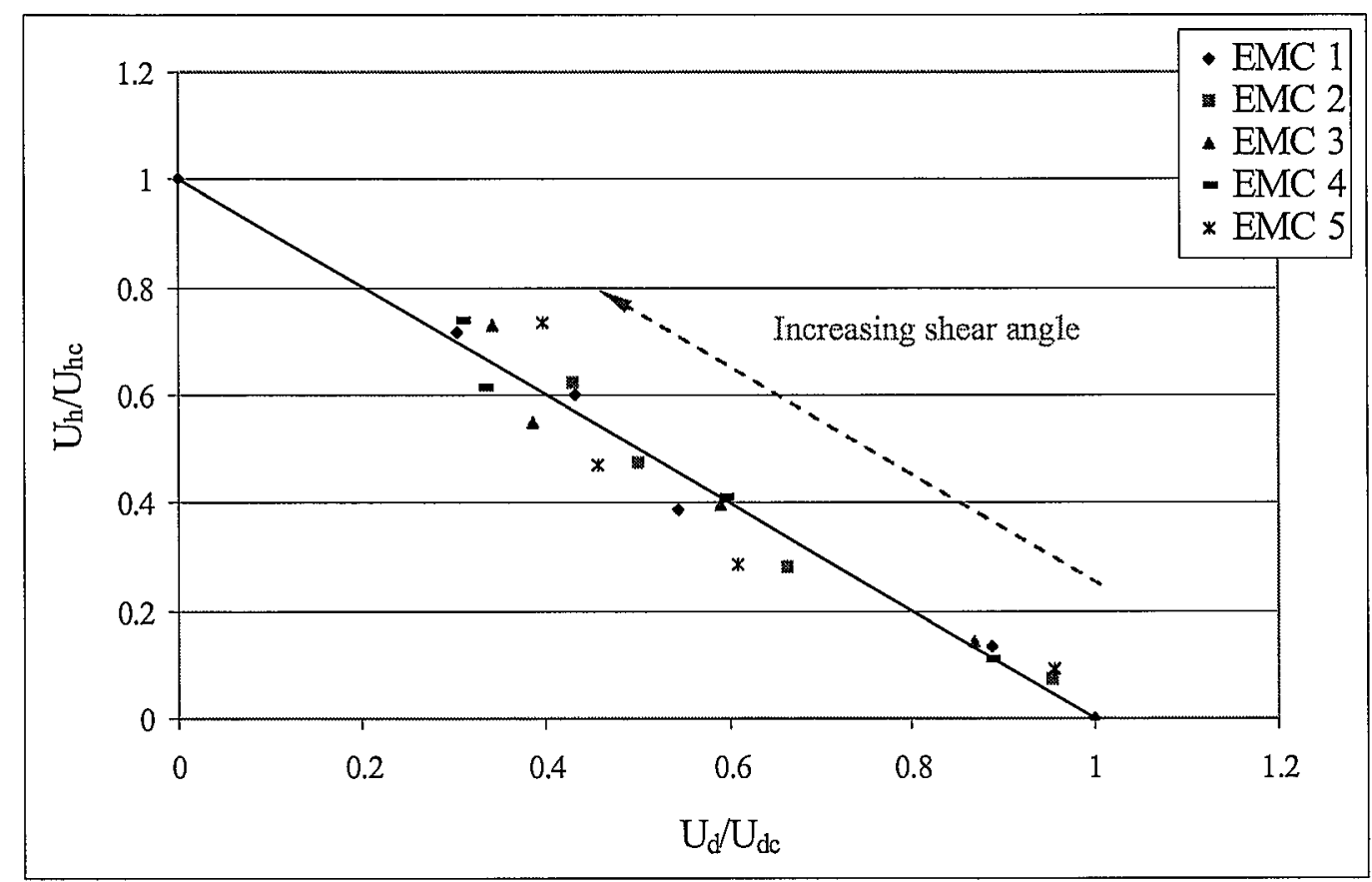

Figure 10. Normalized strain energy densities $U_{d}$ vs $U_{h}$

\section{Discussion}

Based on the linear relation betwee the average interfacial strain energy density, $\bar{U}_{d}$ and $\bar{U}_{h}$, for each kind of EMC material, the unknown critical interfacial strain energy densities, $U_{d c}$ and $U_{h c}$, can be obtained using the interpolation method. Normalized with the allowable interfacial strain energy density for each EMC material, averaged interfacial strain energy densities shown in Fig. 9 can be fitted into a straight line as shown in Fig. 10. This line represents the delamination failure criterion, Equation (5). The failure criterion using the interfacial strain energy density method can simplify the failure prediction of a package design using the button shear test data for material testing to determine the critical average interfacial strain energy density for the selected EMC and copper material system. $U_{d}$ and $U_{h}$ can be calculated in a real package FEA model. If the $U_{d}$ and $U_{h}$ falls outside the safe design region under the curve as defined by the failure criterion of Fig. 10, then interfacial failure will occur in the package. 
Compared to the interfacial failure criterion proposed by Hunt and Carlsson [4], the interfacial strain energy density failure criterion has obvious significant advantages. This method incorporates interfacial stress components into a single parameter interfacial strain energy density, which avoids the difficulty in deciding the delamination initiation point due to different stress component maxima occurring at different locations. Moreover, the energy method conserves the essence of the interfacial bonding which is dependent on surface energy as stated by Kendall [16].

Although interfacial stresses distribution around the loading edge is geometry dependent, the geometry influence is reduced using the averaging method. Hunt and Carlsson [4] and Sung, et al [17] presented the test results with different geometries. In their studies, the average stresses obtained for two different specimens were close to each other showing almost negligible differences. Therefore, the average stresses obtained from different test configurations can be considered almost constant.

\section{Conclusions}

Button shear tests with different shear angles were conducted to evaluate the interfacial strength between EMC and copper leadframe. The averaging approach was used to incorporate a limiting value into a strength-based interfacial strain energy density failure criterion for interfacial delamination. The following results can be summarized:

(1) The failure forces become smaller with angles varying from $-10^{\circ}$ to $20^{\circ}$ for all EMC materials. The negative shear angle gives a more compressive load to the interface, so a higher failure force is needed to shear the button off from the copper substrate. The failure load is inversely proportional to the shear angle.

(2) The average $\bar{U}_{d}$ decreases and $\bar{U}_{h}$ increases with the shear angle varying from $10^{\circ}$ to $20^{\circ}$ and $\bar{U}_{d}$ is nearly linear with $\bar{U}_{h}$ for each EMC material. The 
magnitude of $\bar{U}_{d}$ is higher than that of $\bar{U}_{h}$. It can be concluded that $\bar{U}_{d}$ has more contribution to interface debonding when the shear angle is $-10^{\circ}$ and, on the contrary, $\bar{U}_{h}$ has more contribution to the interface debonding as the shear angle becomes larger.

(3) Based on the experimental results, the unknown allowable interfacial strain energy density for each EMC and copper material system, $U_{d c}$ and $U_{h c}$, can be obtained using the interpolation method. This is used to formulate an interfacial delamination failure criterion. In applying the finite element analysis to a real package model for the determination of the maximum distortional interfacial strain energy density and maximum hydrostatic interfacial strain energy density at the interface, the potential failure across materials interface can be predicted from the interfacial delamination failure criterion.

\section{Acknowledgments}

The project was supported by the Research Grant Council project (HKUST6046/01E) and the authors would like to thank Nitto Denko (HK) Co. Ltd. for supplying the EMC materials.

\section{References}

1. H. Lee and Y. Y. Earmme, IEEE Trans. CPMT, Part A 19, 168-178 (1996).

2. J. C. Brewer and P. A. Lagace, J. Composite Mater.22, 1141-1155 (1988).

3. H. H. Hilton and S. Yi, J. Composite Mater. 27, 1097-1113 (1993).

4. S. Hunt and L. A. Carlsson, ASME Trans. J. Electronic Packaging 115, 249-255(1993).

5. S. Yi, AIAA J. 31, 2320-2328 (1993)

6. G. C. Sih and B. Macdonald, Eng. Fracture Mech., 6, 361-368 (1974). 
7. G. C. Sih and E. P. Chen, Mechanics of Fracture, 6, Martinus Nijhoff Publishers the Hague (1981).

8. G. C. Sih, Mechanics of Fracture Initiation and Propagation, Kluwer Academic Publishers (1991).

9. L. Y. Tong and P. S. Grant, Analysis and Design of Structural Bonded Joints, Kluwer Academic, Boston (1999).

10. H. B. Fan , P. W. K. Chung, M. F. F. Yuen, and P. C. H. Chan, Proc. 52nd IEEE Electronic Component Technology Conference, San Diego, USA, pp.270-276 (2001).

11. H. B. Fan, M. M. F. Yuen, and E. Suhir, Proc. 53th, IEEE Electronic Component Technology Conference, New Orleans, USA, pp. 1160-1164 (2003).

12. H. B. Fan, H. B. Tang, M. M. F. Yuen, and P. C. H. Chan, Proc. 53th, IEEE Electronic Component Technology Conference, New Orleans, USA, pp. 703-707 (2003).

13. M. M. F. Yuen and H. B. Fan, Proc. EuroSIME2003, Aix de Provence, France, pp. 15-21 (2003).

14. T. C. Sun and S. G. Zhou, J. Reinf. Plast. Composites, 7, 515-557 (1998).

15. R, Y, Kim and S. R. Soni, J. Composite. Mater., 18, 70-80 (1984).

16. K. Kendall, Molecular Adhesion and Its Applications: The Sticky Universe, Kluwer Academic / Plenum Publishers (2001).

17. Y. Sung, L. X. Shen, K. K. Iang, and Y. Y. Chee, J. Adhesion Sci. Technol., 14, 93-105 (2000).

18. A. J. Kinloch, Adhesion and Adhesives: Science and Technology, Chapman and Hall (1987).

19. R. D. Adams, J. Adhesion., 30, 219-242 (1989).

20. R. S. Long, Composite Maetr., 25, 391-415 (1991).

21. Q. M. Li, Inte. J. Solids Structures, 38, 6997-7013 (2001). 